Modern Physics Letters B

(C) World Scientific Publishing Company

\title{
OPTIMIZATION OF ROBUSTNESS OF SCALE-FREE NETWORK TO RANDOM AND TARGETED ATTACKS
}

\author{
JIAN-GUO LIU \\ Institute of System Engineering, Dalian University of Technology, 2 Ling Gong Rd., \\ Dalian 116024, P R China \\ liujg004@tom.com \\ ZHONG-TUO WANG \\ Institute of System Engineering, Dalian University of Technology, 2 Ling Gong Rd., Dalian \\ 116024, P R China \\ wangzt@dlut.edu.cn \\ YAN-ZHONG DANG \\ Institute of System Engineering, Dalian University of Technology, 2 Ling Gong Rd., \\ Dalian 116024, P R China \\ yzhdang@dlut.edu.cn \\ Received (8 February 2005) \\ Revised (12 May 2005)
}

\begin{abstract}
The scale-fee networks, having connectivity distribution $P(k) \sim k^{-\alpha}$ (where $k$ is the site connectivity), is very resilient to random failures but fragile to intentional attack. The purpose of this paper is to find the network design guideline which can make the robustness of the network to both random failures and intentional attack maximum while keeping the average connectivity $\langle k\rangle$ per node constant. We find that when $<k>=3$ the robustness of the scale-free networks reach its maximum value if the minimal connectivity $m=1$, but when $\langle k\rangle$ is larger than four, the networks will become more robust to random failures and targeted attacks as the minimal connectivity $m$ gets larger.
\end{abstract}

Keywords: Scale-free network; optimal programme; power-law distribution; random failure.

\section{Introduction}

Recently, there has much interest in the resilience of scale-free network to random attacks or to intentional attacks on the highest degree nodes. ${ }^{1-4}$ Many real-world networks are scale-free and robust to random attacks but vulnerable to intentional attacks. It is important for us to know the optimal scale free network guideline to design networks which are optimally robust against both types of attacks. Although many papers have designed the optimal network topology, such as the two-peak and three-peak optimal complex network 13 , but we can not convert its topology 
to the theoretical optimization directly disobeying its evolutionary principle. On the contrary, we should study the optimal scale-free network guideline to improve the existed scale-free network robustness.

Studies to data 1213 have been considered only the case in which there was only one type of attack in a given network, that is, the network was subject to either a random attack or a targeted attack but not subject to different types of attack simultaneously. A more realistic model is the one in which a network is subjected to simultaneous targeted and random attacks. We focus on the network state after intentional and random attacks which remove fractions $p^{\text {target }}$ and $p^{\text {rand }}$ of the original nodes, respectively.

In the scale-free networks, the degree distribution $P(k)$ is the probability of a node have $k$ connections to other nodes, typically decreases as a power of $k$. Thus with a fraction $p$ of the nodes and their connections of the scale-free network are removed randomly, the random chosen node would have a low degree, so its removal has little effect on the network. Removal of a highly connected node could produce a large effect. However, since such a node may hold significant fractions of the network together by providing connections between many other nodes. Cohen et. al 9 presented a criterion to calculate the percolation critical threshold of randomly connected networks. If we attack the scale-free network intentionally: the removal of sites is not random, but rather sites with the highest connectivity are targeted first. The numerical simulations suggest that scale-free network are highly sensitive to this kind of attack 11 . Cohen et. al ${ }^{10}$ studied the exact value of the critical fraction needed for disruption and the size. Thus networks with a given degree distribution may be very resilient to one type of attack but not to another. This raises two questions we addressed in this paper: How can we optimize scale-free network to both random failure and targeted attack and how to improve the scale free network robustness when the network size becomes larger. If we construct and maintain a network with given number of nodes as being proportional to the average number of links $\langle k\rangle$ per node in the network. Then, our goal becomes how to maximize the robustness of a network with $N$ nodes to both random failure and intentional attack. In our analysis, we compare the robustness of networks which have the same "links" of construction, where we define the cost to be proportional to the average degree $\langle k\rangle$ of all the nodes in the network.

This paper is organized as follows. In the first phase, we give the optimization method to construct the network which is more robust to random failures. In the second phase, we analysis the intentional attack to the scale-free network. In the third phase, we give the optimal strategy of the network design to both random failure and intentional attack.

\section{Optimal Strategy for Random Failures}

Cohen have studied the properties of the percolation phase transition in scale-free random networks, and applied a general criterion for the existence of a spanning 
cluster:

$$
\kappa \equiv \frac{<k^{2}>}{<k>}=2 .
$$

When a fraction $p$ of the nodes are randomly removed, or a fraction $p$ of the links are randomly removed, the distribution of site connectivity is changed from the original $P(k)$ to a new distribution $\widetilde{P}(k)$

$$
\widetilde{P}(k)=\sum_{k_{0} \geq k}^{K} P\left(k_{0}\right)\left(\begin{array}{c}
k_{0} \\
k
\end{array}\right)(1-p)^{k} p^{k_{0}-k} .
$$

Thus, the critical threshold $p_{c}$ can be expressed as: $\frac{\left\langle k_{0}^{2}\right\rangle}{\left\langle k_{0}\right\rangle}\left(1-p_{c}\right)+p_{c}=2$, that is $p_{c}=$ $1-\frac{1}{\kappa_{0}-1}$, where $\kappa_{0} \equiv<k_{0}^{2}>/<k_{0}>$ is calculated from the original connectivity distribution. A wide range of networks have power-law degree distribution: $P(k)=$ $c k^{-\alpha}, \quad k=m, m+1, \ldots, K$, where $k=m$ is the minimal connectivity and $k=K$ is an effective connectivity cutoff presented in finite networks. The parameter $c$ of the power-law distribution can be approximate estimated by $c \approx m^{\alpha-1}(\alpha-1)$. The average connectivity $<k>$ per node is

$$
<k>=\frac{(\alpha-1)}{(\alpha-2)} m\left[1-N^{-\frac{\alpha-2}{\alpha-1}}\right] .
$$

The numerical results show that the exponent $\alpha$ and the minimum connectivity $m$ have following two relationships: (i)To a constant average connectivity $\langle k\rangle$, the exponent $\alpha$ increases when the minimum connectivity $m$ increases; (ii) To the minimum connectivity $m=1$, the exponent $\alpha$ decreases when the average connectivity $<k>$ of the network increases.

The $\kappa_{0}$ of the scale-free network can be approximated by

$$
\kappa_{0}=\frac{2-\alpha}{3-\alpha} \frac{\left[K^{(2-\alpha)}-m^{(2-\alpha)}\right]}{\left[K^{(3-\alpha)}-m^{(3-\alpha)}\right]} .
$$

Our goal is to maximize the threshold for random removal with the condition that the average degree $\langle k\rangle$ per node is constant. We construct the following model.

$$
\left\{\begin{array}{l}
\max \left\{1-\frac{1}{\kappa_{0}-1}\right\} \\
\text { s.t. } \frac{(\alpha-1)}{(\alpha-2)} m\left[1-N^{-\frac{\alpha-2}{\alpha-1}}\right]=<k>, \\
\\
m \in Z^{+}
\end{array}\right.
$$

where $\kappa_{0}=\frac{2-\alpha}{3-\alpha} \frac{\left[K^{(2-\alpha)}-m^{(2-\alpha)}\right]}{\left[K^{(3-\alpha)}-m^{(3-\alpha)}\right]}$. The numerical results suggest that whether the network size $N$ is very large or not, $p_{c}$ reaches its maximum value when $m=1$. We can get the following three conclusions:

(i) If the average connectivity $\langle k\rangle$ per node and the exponent $\alpha$ of the scalefree network is constant, the robustness of the network will decrease when the network size becomes larger. 
(ii) If the network size $N$ is constant, the robustness of the network increases when the average connectivity $<k>$ becomes larger.

(iii) To the random failures, we have to take several times cost to increase the robustness of the scale-free network one percent.

\section{Breakdown under Intentional Attack}

Consider the intentional attack, or sabotage, to the scale-free network, whereby a fraction $p$ of the sites with the highest connectivity is removed, and the links emanating from the sites are removed as well. This would make the cutoff connectivity $K$ of the network reduce to some new value, $\widetilde{K}<K$. Because the upper cutoff $K$ before intentional attack can be estimated from $\sum_{k=K}^{\infty} P(k)=\frac{1}{N}$, the new cutoff $\widetilde{K}$, after the attack, can be estimated by

$$
\sum_{k=\widetilde{K}}^{K} P(k)=\sum_{k=\widetilde{K}}^{\infty} P(k)-\frac{1}{N}=p .
$$

Because $c \approx m^{\alpha-1}(\alpha-1)$, then we have

$$
p \approx\left(\frac{\widetilde{K}}{m}\right)^{(\alpha-1)}-\frac{1}{N} .
$$

If the network size $N$ is very large, $N^{-1} \sim o\left(10^{-2}\right)$, the threshold $p$ for intentional attack may be estimated by $(\widetilde{K} / m)^{(1-\alpha)}=p$.

The intentional attack process to the scale-free network can be described as following two steps:

(i) Removal of the highest connectivity nodes;

(ii) Removal of the links leading to removed nodes.

So intentional attack to the scale-free network can be considered as a random removal of links which connect the removed sites with the remaining sites. We define the probability of removing a link in the sabotage is $\widetilde{p}$ and all links in the network have the same probability of being deleted. We have known that the threshold for random removal of nodes for scale-free network is

$$
1-p_{c}^{\mathrm{rand}}=\frac{1}{\kappa_{0}-1} \text {. }
$$

Then the next task is to find the probability $\widetilde{p}$. The removal of a fraction $p$ of the sites with the highest connectivity results in a random removal of links from the remaining sites that had connected the removed sites with the remaining sites. The probability $\widetilde{p}$ of a link leading to deleted site equals the ratio of the number of links belonging to deleted sites to the total number of links

$$
\widetilde{p}=\sum_{k=\widetilde{K}}^{K} \frac{k P(k)}{<\kappa_{0}>},
$$


Table 1 . The value of $p^{\text {target }}$ when $N=10^{6}$ to different $\langle k\rangle$.

\begin{tabular}{l|cccccc}
\hline & $m=1$ & $m=2$ & $m=3$ & $m=4$ & $m=5$ & $m=6$ \\
\hline$k=3$ & 0.0562 & 0.2010 & - & - & - & - \\
$k=4$ & 0.0627 & 0.2152 & 0.4627 & - & - & - \\
$k=5$ & 0.0617 & 0.2100 & 0.4301 & 0.6240 & - & - \\
$k=6$ & 0.0582 & 0.2003 & 0.4022 & 0.5879 & 0.7172 & - \\
$k=7$ & 0.0523 & 0.1895 & 0.3780 & 0.5567 & 0.6879 & 0.7754 \\
\hline & & & & & &
\end{tabular}

where $\kappa_{0}$ is the initial average connectivity per node. With the continuous approximation, this yields

$$
\begin{aligned}
\widetilde{p} & =\frac{\int_{k=\widetilde{K}}^{K} c k^{1-\alpha} d k}{<\kappa_{0}>}, \\
& =\frac{2-\alpha}{c}\left(K^{2-\alpha}-m^{2-\alpha}\right)^{-1} \frac{c}{2-\alpha}\left(K^{2-\alpha}-\widetilde{K}^{(2-\alpha)}\right) \\
& =\left(K^{2-\alpha}-\widetilde{K}^{(2-\alpha)}\right)\left(K^{2-\alpha}-m^{2-\alpha}\right)^{-1} \\
& =(\widetilde{K} / m)^{2-\alpha}\left[1-\left(\frac{K}{\widetilde{K}}\right)^{2-\alpha}\right]\left[1-\left(\frac{K}{m}\right)^{2-\alpha}\right]^{-1} .
\end{aligned}
$$

In the scale-free network $K>>m$, this yields

$$
\widetilde{p}=(\widetilde{K} / m)^{2-\alpha}\left[1-\left(\frac{K}{\widetilde{K}}\right)^{2-\alpha}\right] .
$$

Replacing $p_{c}$ and $K$ in (7) with (10) and $\widetilde{K}$, this yields the equation:

$$
\left\{1-\left(\frac{\widetilde{K}}{m}\right)^{(2-\alpha)}\left[1-\left(\frac{K}{\widetilde{K}}\right)^{(2-\alpha)}\right]\right\}\left\{\frac{2-\alpha}{3-\alpha} \frac{\widetilde{K}^{(3-\alpha)}-m^{(3-\alpha)}}{\widetilde{K}^{(2-\alpha)}-m^{(2-\alpha)}}-1\right\}=1,
$$

which can be solved numerically to obtain $\widetilde{K}(m, \alpha, K)$, and then $p_{c}(m, \alpha)$ can be retrieved from (6). When $N=10^{6}$, the numerical results are as follows.

From table 1, we can see that: (1) the scale-free network is very fragile when the minimum connectivity $m$ equals to 1 . If intentionally remove about five percent nodes which have the highest connectivity of the scale-free network, the network would collapse. (2) The robustness of the network would increases dramatically when the minimum connectivity $m$ increases.

The maximum value of $p_{c}^{\text {target }}$ is obtained in the situation in which all the nodes have the same degree, in which case the targeted attack becomes equivalent to random failure. Thus we can use the equation (7) to find $p_{c}^{\text {target }}$. The upper bound is therefore given by

$$
p_{c}^{\text {target }} \leq 1-\frac{1}{<k>-1} .
$$

\section{Optimization of Robustness of Complex Network}

When the scale free network was attacks randomly and targeted simultaneously, a metric we can use to measure the robustness of the network to both random failure 
and targeted attack is the sum

$$
p_{c}^{\text {total }}=p_{c}^{\text {rand }}+p_{c}^{\text {target }}
$$

This is only one of a number of possible metrics we can use. The results are, in general, not dependent on the metric chosen.

Our purpose can be stated as follows: for a network of a given number of nodes $N$, how to maximize $p_{c}^{\text {total }}$ while keep the number of links constant.

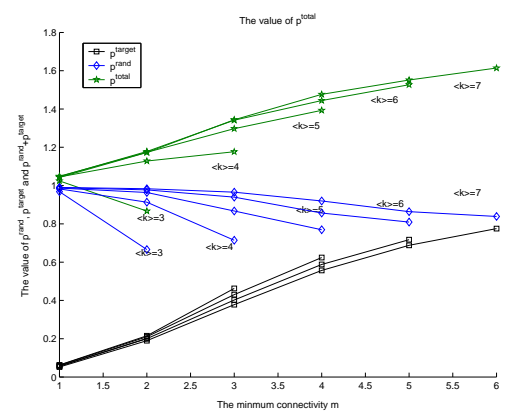

Fig. 1. Random, targeted and total critical percolation thresholds for scale-free networks to different $\langle k\rangle$

In figure 1 , we plot the values of $p_{c}^{\text {target }}, p_{c}^{\text {rand }}$ and $p_{c}^{\text {total }}$ for the minimum connectivity $m$. Because the exponent $\alpha$ would increase as the minimum connectivity increase, we can plot the figure for a range of exponent $\alpha$ too. In the figure, we set the number of nodes $N=10^{6}$ and $k=3,4,5,6,7$. For these choices of $\langle k\rangle$, we find that:

(1) If $m=1$, or $\alpha$ is around 2.5, $p_{c}^{\text {total }}$ is optimized if the average connectivity $<k>=3$. The telephone call graph ${ }^{14,15}$ belongs among this type of network.

(2) If $\langle k\rangle$ is larger than four, the network would become more robust as the minimum connectivity (or the exponent $\alpha$ ) increases. Many networks belong among this type of network, such as coauthorship network. ${ }^{16-21}$ This conclusion shows that the relation between the minimum connectivity nodes of the network is very important. More tight the relationship between the minimum degree nodes of the scale-free networks, more robust the network would be.

\section{Discussion and Summary}

Although the theoretical optimal networks to both random and targeted attacks have been designed, it is very important that some large size networks, such as the internet, is a self-organizing system, evolve and drastically changes over time according to evolutionary principle dictated by the interplay between cooperation and 
competition. To the exist growing scale-free network, we can not convert its topology into the theoretical optimization directly disobeying its evolutionary principle. But we can improve the network robustness. Firstly, we must know the optimal guideline to maximum the scale-free network robustness.

The $p_{c}^{\text {total }}$ characterizes the robustness of the scale-free network to random failures and intentional attacks simultaneously. In this paper we analyze the scale-free network robustness to both random failures and intentional attacks simultaneously and find that the minimum degree of the network is very important to the scale-free network robustness with a constant $\langle k\rangle$. To an exist growing scale-free network, if the network average degree per node is around three, we should keep its minimum degree around one to improve the network robustness. But if the network average connectivity is larger than four, we should add its minimum degree to improve its robustness to both attacks, which mean that the relationship between the minimum connectivity nodes would become very important for the network robustness.

\section{Acknowledgments}

This research was supported by the Chinese Natural Science Foundation (Grant No. 70431001, 70271046).

\section{References}

1. R. Albert and A.-L. Barabási, Rev. Mod. Phys. 74, 47 (2002).

2. M. E. J. Newmann, SIAM Rev. 45, 167 (2003).

3. J. F. F. Mendes, S. N. Dorogovtsev and A. F. Ioffe, Evolution of Networks: From Biological Nets to the Internet and the $W W W$ (Oxford University Press, Oxford, 2003).

4. R. Pastor-Satorras and A. Vespignani, Evolution and Structure of the Internet: A Statistical Physics Approach (Cambridge University Press, Cambridge, 2004).

5. P. Erdŏs and A. Rényi, Publicationes Mathematicae 6, 290 (1959).

6. P. Erdŏs and A. Rényi, Publicationes Mathematical Inst. of the Hungarian Acad. of Sciences 5, 17 (1960).

7. D. J. Watts and S. H. Strogatz, Nature 393, 440 (1998).

8. B. Bollobas, Random Graphs (Academic, London, 1985).

9. G. Paul, T. Tanizawa, S. Havlin and H. E. Stanley, Eur. Phys. J. B 38, 187 (2004).

10. R. Cohen, K, Erez, D. ben-Avraham, S. Havlin, Phys. Rev. Lett. 85, 4626 (2000).

11. R. Cohen, K, Erez, D. ben-Avraham, S. Havlin, Phys. Rev. Lett. 86, 3682 (2001).

12. R. Albert, H. Jeong, and A. L. Barabási, Nature, 406, 6794 (2000).

13. A. Valente, A. Sarker, and H. A. Stone, Phys. Rev. Lett. 92, 118702 (2004).

14. G. Paul, T. Tanizawa, S. Havlin, and H. E. Stanley, Eur. Phys. J. B 38, 187 (2004).

15. W. Aiello, F. Chung, and L. Lu, In Proceedings of the 32nd Annual ACM Symposium on Theory of Computing, Association of Computing Machinery, New York, 171 (2000).

16. W. Aiello, F. Chung, and L. Lu, In Handbook of Massive Data Sets, J. Abello, P. M. Pardalos, and M. G. C. Resende, eds., Kluwer Academic, Dordrecht, 97 (2002).

17. G. F. Davis, M. Yoo, and W. E. Baker, University of Michigan Business School, Ann Arbor, $M I$ (2001).

18. M. E. J. Newman, S. H. Strogatz, and D. J. Watts, Phys. Rev. E, 64, 026118 (2001).

19. R. de Castro and J. W. Grossman, Math. Intelligencer, 21, 51 (1999).

20. J. W. Grossman and P. D. F. Ion, Congr. Numer., 108, 129 (1995). 
July 20, 2018 19:51 WSPC/INSTRUCTION FILE ws-mplb050699

8 Jian-Guo Liu

21. M. E. J. Newman, Phys. Rev. E, 64, 016131 (2001).

22. M. E. J. Newman, Proc. Natl. Acad. Sci. USA, 98, 404 (2001). 